\title{
Protective sensation in the foot in leprosy
}

\author{
C J HAMMOND \& P KLENERMAN \\ Oxford University Medical School, Osler House, John Radcliffe \\ Hospital, Oxford OX3 9DU
}

Accepted for publication 22 June 1988

\begin{abstract}
Summary Plantar ulceration is a significant problem in leprosy patients and accounts for a large proportion of hospital admissions. Three methods of sensory testing were employed to see if a high-risk group of people with loss of protective sensation might be selected, so that the serious first ulcer might be prevented. Three groups of patients were studied: 41 leprosy patients with ulcers, 41 without ulcers and 48 control subjects without leprosy. The results show that either or both Semmes-Weinstein nylon monofilaments and biosthesiometer may prove a more reliable method of sensory testing than the standard WHO pencil stimulus.
\end{abstract}

\section{Introduction}

Plantar ulceration resulting from damage to the anaesthetic foot is a major problem in the management of leprosy patients. Patients with ulcers form a large proportion of those admitted to leprosy hospitals, and these admissions can cause loss of income as well as further physical disability and social isolation for the patients. Loss of 'protective sensation' is a major factor in ulcer formation, which will in turn lead to a scarred foot, even more prone to further ulceration. Therefore, prevention of the first ulcer must be a priority in any leprosy programme.

The strategy for prevention is primarily education of the patient about care of insensitive feet, and the issue of protective footwear such as microcellular rubber (MCR) sandals. With limited resources, the clinician needs to define a high-risk group quickly and easily, in order to concentrate attention on these individuals.

The purpose of this study was to assess two techniques of defining such a group at risk of plantar ulceration. A vibration meter or 'biothesiometer' (Figure 1) has been used in detecting early loss of vibration sensation in diabetic patients in the $\mathrm{UK}^{1}$ but there is only one report of its use in the leprosy field. ${ }^{2}$ The second method was evaluation of pressure sensation using a graded range of nylon monofilaments (Semmes-Weinstein filaments, Figure 2) which have been useful in assessment of mild nerve damage. ${ }^{3}$ These monofilaments have been used in research on plantar sensation in patients with leprosy in America ${ }^{4}$ but have not been assessed in barefoot populations in the Third World.

The aim has been to compare these two methods of assessment, together with the standard WHO test, and to define a point at which loss of 'protective sensation' may be said to occur. 


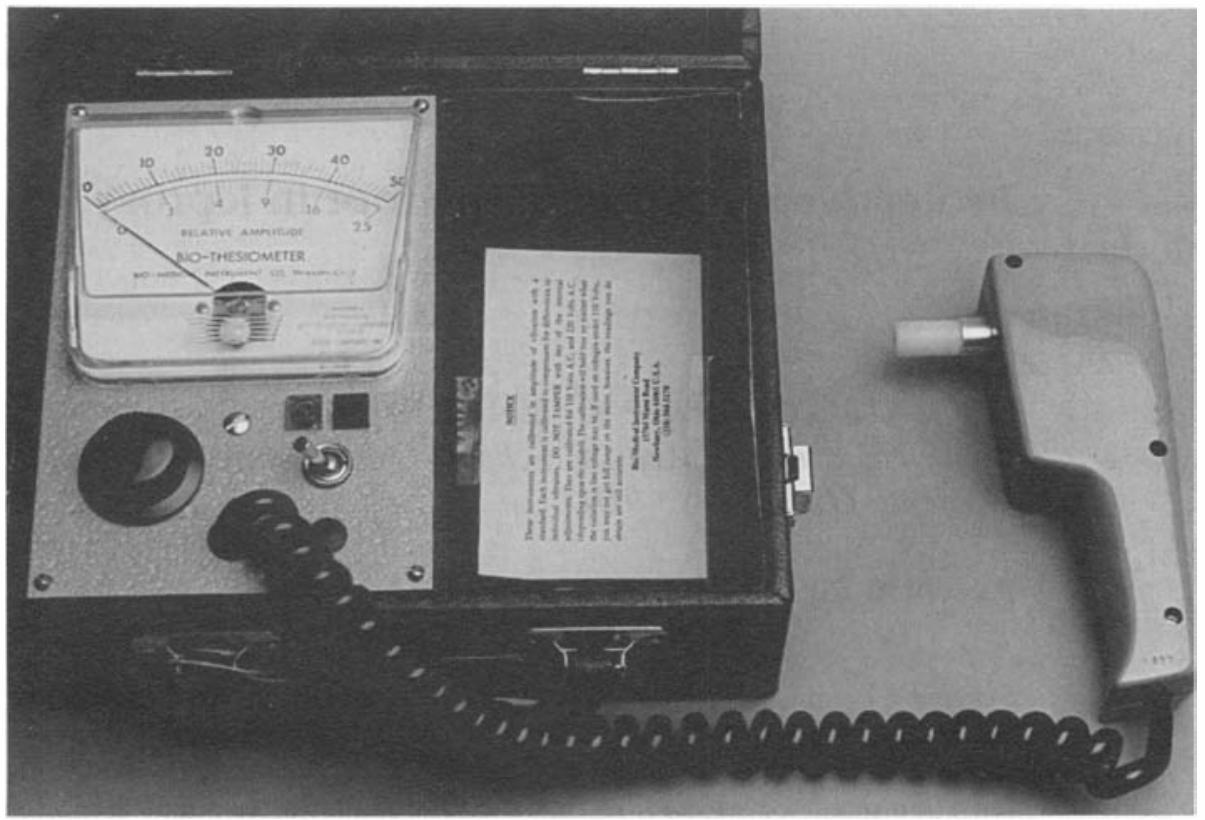

Figure 1. Biothesiometer.

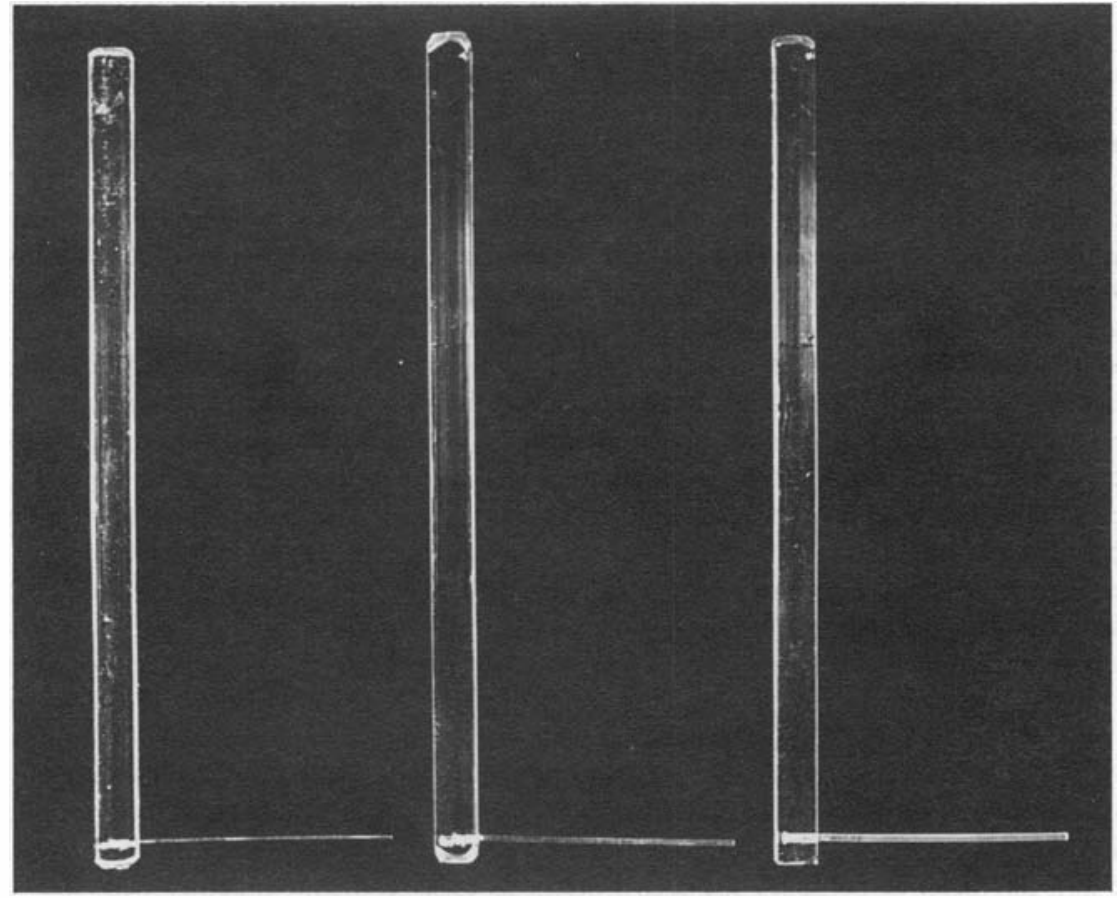

Figure 2. Semmes-Weinstein monofilament. 


\section{Materials and methods}

Three groups of patients were considered: leprosy patients with plantar ulcers, leprosy patients without ulcers, and a control group without leprosy, all matched for age and sex (see Table 1). The leprosy patients were drawn from in-patients and out-patients at the Dr Bandorawalla Leprosy Hospital, near Pune, India, and the controls were local agricultural workers and non-leprosy patients at the Dermatology Clinic at Sassoon Hospital in Pune. Excluded from all 3 groups were those patients with known diabetes mellitus, any other foot pathology, and those unable to understand the test (of whom there were 4). The group with ulcers included all those with a past history of or present plantar ulceration, but no clinical signs of tarsal disintegration (included in a separate study). The non-ulcered group excluded any leprosy patient with a past history or suspicion of ulcer, and also any patient in lepra reaction.

Materials used were: 1 set of 20 Semmes-Weinstein graded nylon monofilaments (Research Designs Inc.), consisting of monofilaments or 'hairs' range numbers 2.84 to 6.65 (these numbers relate to the logarithm of the force required to bend the hair); 1 biosthesiometer delivering a vibrational stimulus at a fixed frequency of $120 \mathrm{~Hz}$ and of varying amplitude from 0 to $25 \mu \mathrm{m}$ (Bio-Medical Instrument Co., Ohio, USA); a 2B pencil used for WHO standard sensory testing.

\section{Method}

Patients were tested in a quiet room, with the aid of a translator. Details of the patient's age, sex, history of past and present ulceration and any history of operations were noted. Disease type, duration and treatment were also recorded.

After adequate explanation and demonstration, the patient's sensory threshold using the nylon filaments was determined at 3 sites; great toe pulp, first and fif th metatarsal heads. The method is described elsewhere, ${ }^{5}$ and the threshold was taken as that filament at which the subject could accurately and reproducibly detect the site of pressure stimulus. The highest threshold from the 3 sites was taken as the threshold for that foot.

Standard sensory testing as recommended by $\mathrm{WHO},{ }^{6}$ using pressure from a pencil point at the 3 sites was assessed. A 'positive' WHO test was taken as the ability to point accurately to the site of dimpling at all 3 positions, a "negative" test implying inability to recognize one or more positions.

Vibration sensation was tested at 2 sites, the great toe pulp and the medial malleolus, as described. ${ }^{7}$ The vibrating rod was applied at each site with constant light pressure and the amplitude of vibration slowly increased until the subject first noticed the sensation. This was repeated 3 times at each site and the mean amplitude calculated. The sum for each foot was recorded from the means at the 2 sites, with those subjects unable to feel a maximum vibration being given an arbitrary value of $25 \mu$.

Table 1. Comparison of groups.

\begin{tabular}{lccc}
\hline & & $\begin{array}{c}\text { Patients } \\
\text { Controls }\end{array}$ & $\begin{array}{c}\text { Patients } \\
\text { with ulcers }\end{array}$ \\
\hline Number of patients & 48 & 41 & 41 \\
Number of feet measured & 96 & 82 & $59^{*}$ \\
Age range & $13-55$ & $15-54$ & $12-55$ \\
Mean age (years) & $32 \cdot 7$ & $31 \cdot 7$ & $32 \cdot 4$ \\
Sex ratio & $32: 16$ & $31: 10$ & $31: 10$ \\
\hline
\end{tabular}

\footnotetext{
* 18 subjects had bilateral ulcers
} 


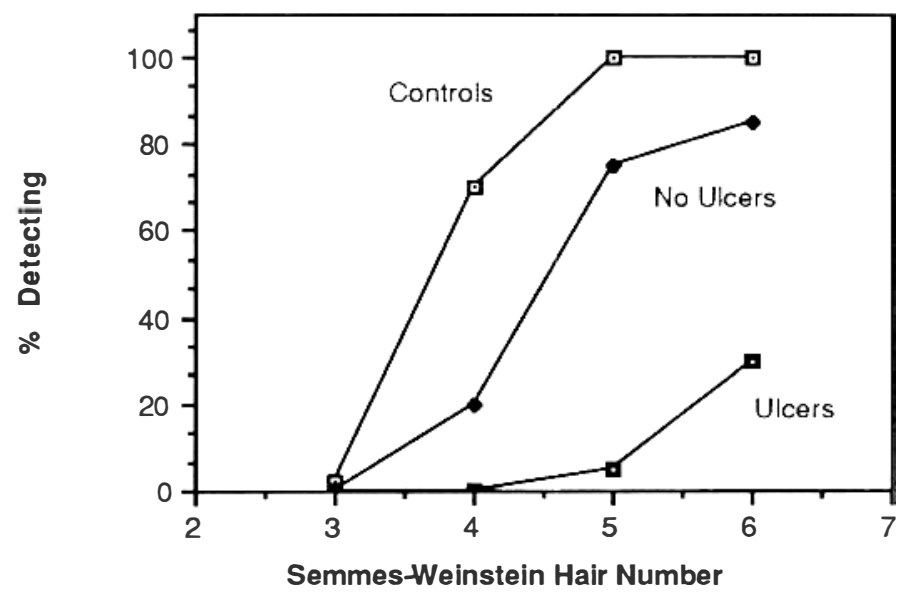

Figure 3. Distribution of pressure sensory thresholds in feet of patients and controls.

\section{Results}

The composition of the 3 groups (patients with ulcers, patients without ulcers and controls) are shown in Table 1.130 subjects were measured, 41 in the ulcered group, 41 in the non-ulcered group, and 48 controls. Fifty-nine feet with ulcers were examined.

Figure 3 shows the cumulative frequency curvesf or these 3 groups, of sensation threshold when tested with Semmes-Weinstein monofilaments, i.e. the proportion of patients able to feel each filament. Considering the threshold value represented by the 5.07 filament, this could be detected by $99 \%$ of the control feet, by $70 \%$ of feet of leprosy patients without ulcers, and only $5 \%$ of feet of patients with ulcers. (Differences significant at $p<0 \cdot 01$ using chi-squared test).

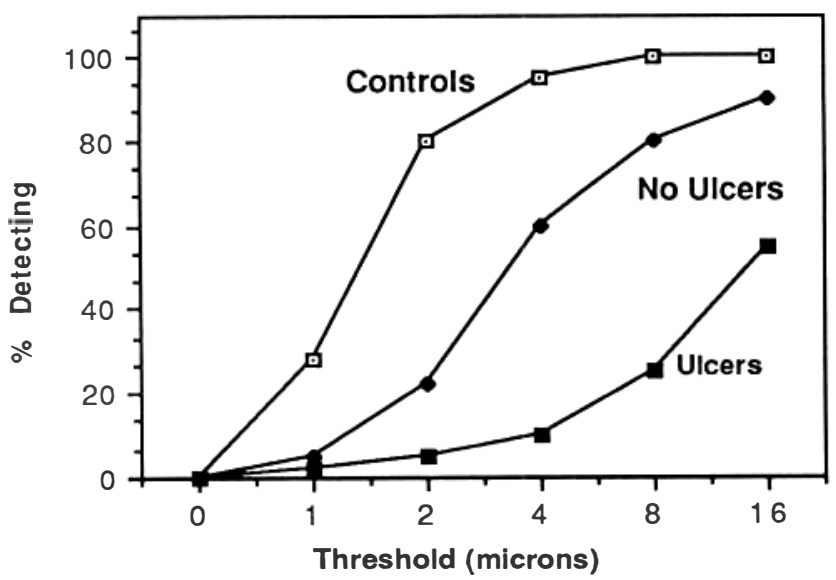

Figure 4. Distribution of vibration sensory thresholds in feet of patients and controls. 


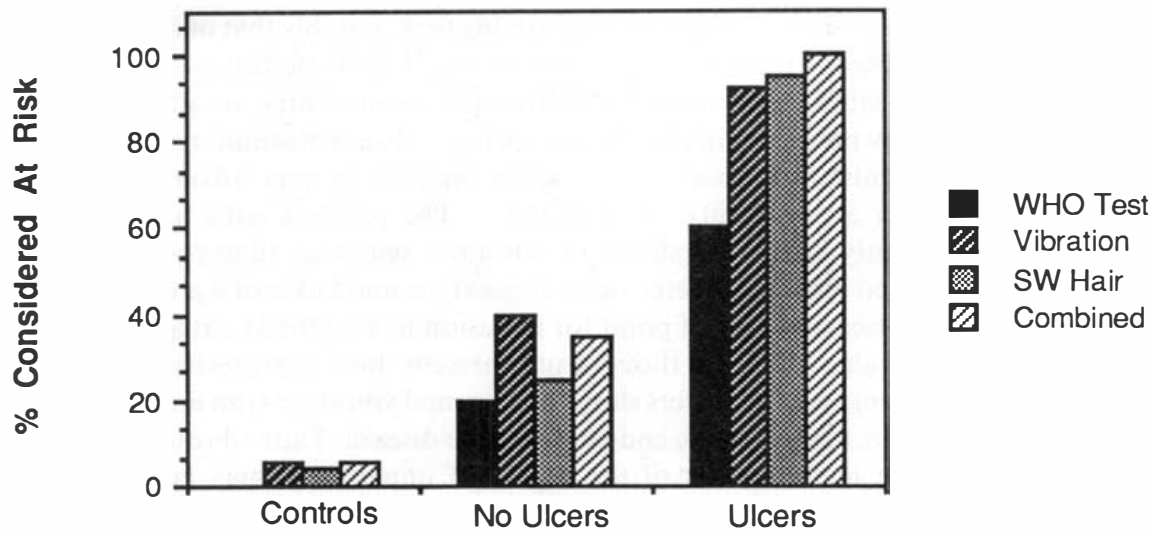

Figure 5. Percentages of patients considered to have lost protective sensation by four criteria.

The cumulative frequencies of vibration threshold are demonstrated in Figure 4 for the 3 groups. Considering a threshold value for a foot of $4 \mu$ this could be detected by $99 \%$ of normals. $60 \%$ of non-ulcered patients, and $10 \%$ of patients with ulcers. (Differences significant at $p<0.01$ using the chi-squared test.)

Sixty-one per cent of patients with ulcers were unable to detect the pencil stimulus as described, and these results are considered in comparison to the other methods of testing in figure 5.

\section{Discussion}

The concept that loss of a certain level of 'protective sensation' in anaesthetic feet puts them at risk of plantar ulceration is widely used. ${ }^{4}$ The aim of this study was to define this level in patients with leprosy, and to do so using simple clinical tools which might be applicable in a First or Third World hospital. In the past a battery of tests has been proposed in order to evaluate peripheral neuropathy in leprosy patients, ${ }^{8}$ including the use of graded nylon filaments and optional low frequency $(60 \mathrm{~Hz})$ and high frequency $(250 \mathrm{~Hz})$ tuning forks. It was thus of further interest to compare different modalities of sensory testing in the same patients.

Anaesthesia is not the only important factor in the aetiology of plantar ulceration in leprosy. Nerve damage leads to loss of autonomic supply and hence drying of skin, and motor damage can result in undue pressures on certain parts of the sole, e.g. through drop foot. ${ }^{9}$ Another extremely important factor is the patient's health education and subsequent personal foot care and use of protective shoes. Thus in the group without ulcers there is a proportion of patients who have lost protective sensation and so are still at high risk of ulceration. Indeed it is exactly these patients whom one wishes to identify, before the first ulcer has developed. ${ }^{10}$

\section{BIOSTHESIOMETER}

Although the biosthesiometer has been in use for over 40 years, it received little attention in the UK before the early 1980s. Since then, it has been used in the study of peripheral neuropathy in diabetic patients (another group susceptible to plantar ulceration) in whom the problems of anaesthesia are compounded by the vascular complications of that disease. ${ }^{11}$ The vibrometer or biosthesiometer has 
a number of advantages over clinical testing using a tuning fork, notably that of quantification ${ }^{12}$ and normal values are available for the UK population for use in diabetic clinics. ${ }^{7}$

In contrast, testing of vibration sensation in leprosy has received little attention and it has been thought that it is relatively preserved in this disease, being a 'deep sensation' namely 'position and vibration sensation are entirely normal except in the unusual or very advanced case in which multiple major nerves in an extremity are affected. ${ }^{13}$ The patients with ulcers in this study demonstrated a significantly higher threshold for vibration sensation than controls and patients without ulcers. A combined biosthesiometer value at great toe and ankle of $4 \mu$ would identify $90 \%$ of patients with ulcers if used as a cut-off point for inclusion in a high-risk category (see Figure 5), and readings above this value would be thought to represent 'loss of protective sensation' in this disease. That $40 \%$ of patients without ulcers showed abnormal vibration is an indication that loss of vibration sensation is not restricted to the end-stages of the disease. Thus vibration sense in leprosy is not only impaired, but measurement of the degree of impairment may be of some value in distinguishing patients at risk of ulceration.

Advantages of the use of a biosthesiometer in this situation are that it is an easily reproducible test, and relatively easy for the patients to understand. In practical terms it is expensive and requires an electrical supply which is not always available in the Third World clinic. A further disadvantage is the increase in normal thresholds, both mean and variance, with age, ${ }^{7}$ thus limiting its usefulness over the age of 60 . However, out of a potential population of over 80 patients with ulcers, only 2 were over 60 year old, and this would not appear to be a serious practical limitation in leprosy work. Other potential limitations have been pointed out by Williams et al. in a recent paper. ${ }^{14}$

\section{SEMMES-WEINSTEIN MONOFILAMENTS}

Nylon filaments have been previously recommended in the identification of sensory neuropathy in leprosy ${ }^{8}$ and have been used in the United States to define a minimum sensory level in patients with ulcers. ${ }^{4}$ It was suggested that similar experiments should be performed in the Third World, where barefoot walking is common and normal values may be different. Our results provide further evidence that such testing is of value in identifying loss of 'protective sensation'. Use of a threshold value of $5.07(11.8 \mathrm{~g})$ would identify $95 \%$ of patients with ulcers if used as a minimum cut-off point foridentification of a high-risk group, and by this criterion $24 \%$ of patients without ulcers would be in such a category. These results agree with those of Birke \& Sims, ${ }^{4}$ who also proposed a 5.07 filament for this purpose. It is interesting to note that the average value of our control population (4-11) does not differ significantly from their normal quoted value (4-17), despite the fact that the majority of controls were accustomed to barefoot walking. Loss of vibration sensation accompanies loss of pressure sensation, a correlation first suggested by Von Frey in his seminal work on the subject. ${ }^{14}$

Although use of a set of monofilaments is a slow and expensive method of testing, use of a single filament such as the 5.07 hair as a proposed discriminatory test is quick, and it is possible to make such individual filaments. ${ }^{8}$ One disadvantage of this method of testing is that the patients find it relatively difficult to understand, and care must be taken to avoid 'cheating'. The force exerted by a filament is thought to change with a variety of factors including temperature and possibly wear, so regular recalibration may be required. It has been pointed out that the monofilaments are simple to use but easy to misinterpret. ${ }^{16}$

\section{WHO STANDARD METHOD}

The WHO-recommended method of testing has the advantage of being quick, simple and cheap, but lacks the reproducibility of either of the above methods. Although a pencil was used in this study, many centres use a ballpoint pen which has a more constant tip, therefore minimizing variation in pressure with a constant force. 
In this series only $61 \%$ of patients with ulcers would be said to have lost protective sensation by this criterion, and so a significant proportion of such patients would not have been included in a high-risk group. In many ways, use of a single hair may be regarded as a more refined version of this test, with greater sensitivity and reproducibility.

\section{COMPARISON OF METHODS}

Four methods of identifying high-risk patients are compared in Figure 5-5.07 monofilament alone, biosthesiometer alone, WHO test alone and filament and biosthesiometer combined. Use of the last method would identify $100 \%$ of patients with ulcers as belonging to the high-risk category, while use of a 5.07 filament alone would identify $95 \%$ of patients and could thus provide a useful screening test in a Third World clinic. An additional $24 \%$ of patients without ulceration would have lost protective sensation by this criterion.

Since this is a retrospective study it is not possible to give predictions of risk on the basis of this data, but as such it might form the basis of a prospective investigation.

\section{Conclusion}

It has been proposed that measurement of vibration sensation may be a usef ul clinical tool in the assessment of the insensitive foot in leprosy, even in the relatively early stages of the disease. The use of a single monofilament either alone or in combination with biosthesiometry might prove a reliable method for identifying patients at high risk of plantar ulceration in a leprosy clinic. For this purpose, a 5.07 monofilament would appear to be the most appropriate.

It should, however, be emphasized that simply identifying a patient as 'high-risk' is only a small part of ulcer prevention. Much work needs to be done before this stage in early detection and treatment of leprosy, and much remains to be done afterwards, for effective patient education in foot care and lifelong footwear is an all too often neglected part of leprosy management.

\section{Acknowledgments}

We would like to thank all the staff at the Dr Bandorawalla Leprosy Hospital, Pune, its Hon. President Dr Jal Mehta, and Dr Sunil Tolut, Dermatology Dept., Sassoon Hospital, Pune, for their help and support with this project. We are indebted to Mr Anjan Dei for his help with translation. We would also like to thank Dr Colin McDougall and Professor L Klenerman for providing us with ideas and equipment.

We are grateful for grants from LEPRA, Journal of Bone and Joint Surgery, St Francis Leprosy Guild, Medical Defence Union, Anglo-American Corporation (Zimbabwe) Ltd, and Oxford University Medical School.

\section{References}

${ }^{1}$ Hillson RM, Hockaday TDR, Newton DJ. Hypoglycaemia is one correlate of deterioration in vibration sense during the five years after diagnosis of type II (non-insulin dependent) diabetes. Diabetologica, 1984; 26: $122-6$.

2 Theriot S et al. Reliability of the biosthesiometer in measuring plantar vibratory thresholds. Int J Lepr, 1987; 55: 416.

3 Naafs B, Dagne T. Sensory testing; a sensitive method in the follow-up of nerve involvement. Int J Lepr, 1977; 45: 364-8.

${ }^{4}$ Birke JA, Sims DS. Plantar sensory threshold in the ulcerative foot. Lepr Rev, 1986; 57: 261-7. 
${ }^{5}$ Bell-Krotowski J, Tomancik E. The repeatability of testing with Semmes-Weinstein monofilaments. $J$ Hand Surg, 1987; 12A: 155-61.

${ }^{6}$ WHO Expert Committee on Leprosy. 4th Report. WHO Technical Report Series, 1976: 77.

7 Bloom S et al. Use of a biosthesiometer to measure individual vibration thresholds and their variation in 519 non-diabetic subjects. $\mathrm{Br} \mathrm{Med} J, 1984$; 288: 1793-5.

8 Pearson JM. The evaluation of nerve damage in leprosy. Lepr Rev, 1982; 53: 119-30.

${ }^{9}$ Brand PW. The insensitive foot. In: Disorders of the foot. Jahss M (ed.). Philadelphia: WB Saunders, 1982; 1266-86.

10 Brand PW. Deformity in Leprosy. In: Leprosy in Theory and Practice. Cochrane RF, Davey TF (eds), Bristol: John Wright and Sons, 1964: 447.

11 Edmonds ME. The diabetic foot: pathophysiology and treatment. Clin Endo Metab, 1986; 15(4): 889-99.

12 Gregg EC. Absolute measurement of vibratory threshold. AM A Arch Neurol and Psych, 1951; 66(4): 403-11.

13 Dyck Thomas, Lambert, Bunge (eds). Peripheral Neuropathy, 2nd ed. Philadelphia: WB Saunders, 1984: vol 2; 1963

14 Williams G et al. variability in vibration perception threshold among sites: a potential source of error in biosthesiometry. Br Med J, 1988; 296: 233-5.

15 Von Frey, Physiologische Versuche uber das Vibrationsgef uhl. 1915. Quoted in Fox JC and Klemperer W. Arch Neurol Psychiat, 1942; 48: 622-45.

16 Levin S et al. Von Frey's method of measuring pressure sensibility in the hand: an engineering analysis of the Weinstein-Semmes pressure aesthesiometer. J Hand Surg, 1978; 3: 211-16. 